\title{
Perspectivas de Design Participativo Utilizando Brain Draw
}

\author{
Rallyson dos S. Ferreira' ${ }^{1}$, Thaís H. Chaves de Castro ${ }^{1}$ \\ ${ }^{1}$ Instituto de Computação (IComp) - Universidade Federal do Amazonas (UFAM) \\ Caixa Postal 15.064 - 91.501-970 - Manaus - AM - Brasil \\ rallysonferreira@ufam.edu.br, thaiseicomp.edu.br
}

\begin{abstract}
This paper deals with the application of the Brain Draw technique, used in participatory design, adapted and contextualized to increase the communication possibilities of children with autism when expressing their preferences regarding shared study space settings. In this perspective, using inclusive design models, we foster the participation of those children during all design phases. However, there are two main difficulties: the first one is that when it comes to children, you have to use immediate feedback activities; the other one is that being children with autism, they still have difficulties expressing themselves. The results are discussed to prospect future perspectives of evolution and use of the approach for other contexts.
\end{abstract}

Resumo. Este artigo aborda a aplicação da técnica Brain Draw, utilizada em design participativo, adaptada e contextualizada para aumentar as possibilidades de comunicação de crianças com autismo ao expressarem suas preferências com relação a contextos de espaços compartilhados de estudo. Nesta perspectiva, usando modelos de design inclusivo, incentivamos a participação dessas crianças, durante todas as fases do design. No entanto, há duas dificuldades principais: a primeira é que, se tratando de crianças é necessário oferecer atividades de feedback imediato; a outra é que sendo autistas, essas crianças possuem dificuldade de expressão. Os resultados são discutidos para prospectar perspectivas futuras de evolução e uso da abordagem para outros contextos.

\section{Introdução}

Embora a literatura demonstre muitas iniciativas sobre o processo de Design Participativo (DP) e Design Inclusivo (DI) poucos trabalhos mostram o envolvimento de crianças dentro desse contexto, cabendo a responsabilidade, quase que exclusivamente, aos designers, que se baseia somente na coleta inicial dos requisitos [Nunes, Luz e Lemos 2016].

Apesar de, inicialmente, o DP ter sido usado no contexto de design baseado em grupos [Muller e Kuhn 1993] sua aplicação no desenvolvimento de jogos educacionais voltados para crianças nas séries iniciais é bastante singular e exige a participação direta das mesmas [Ismail e Ibrahim 2017]. Pesquisas reforçam a necessidade do DP para compreender e analisar dados obtidos das fases iniciais do design como imersão, análise e síntese, e envolvendo crianças com transtornos de neurodesenvolvimento na infância, como é o caso de crianças com autismo [Satterfield e Fabri 2017].

Algumas pesquisas utilizam métodos e técnicas para observação e percepção, a fim de analisar as escolhas das crianças com autismo durante a utilização das aplicações [Wilson et al 2017], [Spiel e Malinverni 2017]. Dessa forma, a técnica conhecida como 
"Brain Draw" vem demonstrando resultados promissores baseado nessas perspectivas. Tal técnica visa obter representações das crianças por meio de desenhos em que as mesmas sublinham os traços para formar imagens e assim identificar através de expressões suas preferências [Melo, Baranauskas e Soares 2008].

Portanto, neste artigo embutimos a técnica em uma aplicação mobile denominada Drawing Kids. Além de a aplicação apresentar as mesmas características da técnica em si (oferecendo espaço de desenho virtuais) possui outras funcionalidades a fim de obter das crianças informações relevantes sobre suas habilidades espaciais e suas predileções em relação à organização de seu espaço de aprendizagem. Essas funcionalidades, desenvolvidas inicialmente para crianças com autismo, passaram por um processo de avaliação tornando-a acessível e reforçando a análise da aplicação junto aos profissionais de apoio (professores, cuidadores, mediadores, psicólogos, etc).

O resultado dos testes com a aplicação fornece uma maneira alternativa para introduzir crianças com transtornos de neurodesenvolvimento, especificamente crianças com autismo, no processo de design, identificando suas diferentes formas de expressão e habilidades através da utilização de objetos virtuais que são representações dos reais presentes em seus contextos. Além de auxiliar na expressão das crianças, a técnica Brain Draw baseada na aplicação Drawing Kids auxilia os designers a compreender e adaptar novas ferramentas computacionais a características individuais das pessoas.

\section{Background}

\subsection{Design Participativo e Brain Draw}

O DP é considerado um dos melhores métodos para entender as necessidades de um grupo de pessoas e criar soluções de alta qualidade e bem projetadas para atender às suas necessidades. No entanto, o envolvimento de pessoas com deficiências cognitivas em diversos processos de design, como no processo de design de experiência do usuário, do inglês (User eXperience Design - UXD), pode ser difícil em um ambiente educacional. Em [Satterfield e Fabri 2017] é evidenciado que frequentemente, crianças com autismo são excluídas da coleta de dados do UXD devido à baixa de capacidade cognitiva e habilidades linguísticas para participar de maneira significativa.

Como uma das motivações para a adoção de uma abordagem participativa é que dessa forma a capacidade de reconhecer as características e as necessidades das pessoas a quem o design se destina aumenta, podendo assim ter foco nas reais necessidades dos principais interessados, como também na inovação e no planejamento mais consistente. As inúmeras perspectivas, para os quais as tecnologias são projetadas, tornaram o DP um tema central na cultura e prática da pesquisa em Interação Humano Computador (IHC), particularmente no design de interação [Frauenberger, Makhaeva e Spiel 2017].

As tecnologias desenvolvidas para apoiar crianças com autismo tendem a usar conteúdos pré-definidos para aprimorar habilidades específicas, como comunicação verbal ou reconhecimento de emoções. Poucos trabalhos mobilizam os pontos fortes e capacidades da criança (geralmente muito específicos). As tecnologias digitais oferecem oportunidades para que as crianças personalizem o aprendizado com seu próprio conteúdo, seguindo seus próprios interesses e possibilitando sua autoexpressão. Os professores podem desempenhar um papel fundamental na apropriação do design na 
sala de aula, especificamente por meio do conhecimento profundo de cada criança e de suas necessidades, pontos fortes e interesses individuais [Wilson et al 2017].

Nessa perspectiva, a técnica conhecida como "Brain Drawn" pode auxiliar os profissionais da educação desempenhando a tarefa de reconhecer as percepções de crianças com autismo através de uma aplicação que busca a interação de forma individual e autônoma [Melo, Baranauskas e Soares 2008]. Dessa forma, com o Drawing Kids, conseguimos discutir, de forma mais detalhada e específica, as preferências de design de crianças com autismo no contexto escolar.

\subsection{Transtorno do Espectro Autista e Aprendizagem}

O Transtorno do Espectro Autista (TEA) consiste na presença de um desenvolvimento comprometido ou acentuadamente anormal da interação social e da comunicação e um repertório muito restrito de atividades e interesses. As manifestações do transtorno variam, dependendo do nível de desenvolvimento e da idade cronológica [Santana et al 2017]. Os sintomas do TEA costumam aparecer antes dos 3 primeiros anos de vida, a síndrome é caracterizada pela deficiência em atividades, comunicação, linguagem e interação social, podendo ocorrer a ausência de respostas a sentimentos de outras pessoas, bem como a falta de modulação de seus próprios comportamentos.

O TEA apresenta sintomas que causam, frequentemente, prejuízos para as atividades diárias, inclusive nas atividades escolares, uma vez que não se consegue lidar com a variedade de estímulos. Muitas pessoas com TEA também apresentam comprometimento intelectual e/ou da linguagem, além de comprometimentos motores, que vão de leves dificuldades de coordenação a graves [APA 2014].

Nas últimas décadas, tecnologias computacionais, especialmente relacionadas à hipermídia e $W e b$, têm sido utilizadas como ferramentas de apoio para crianças com TEA, bem como para seus pais, terapeutas e educadores [Britto e Pizzolato 2016]. Soluções de hipermídia educacional projetadas adequadamente para autistas permitem que a tecnologia seja utilizada de forma benéfica para intervenção pedagógica ou terapêutica [Silva, Salgado e Raposo 2013]. Sabe-se também que crianças com autismo usualmente têm alta preferência por uso de computadores e tablets, o que pode facilitar a introdução desses dispositivos dentro ambiente escolar [Britto e Pizzolato 2016].

\subsection{Crianças com Autismo no DP}

O DP é uma metodologia bastante conceituada em IHC (Interação HumanoComputador) que busca interpretar e adaptar ferramentas computacionais às características de cada pessoa. No entanto, devido a impossibilidade de envolver crianças com autismo no processo de design é necessário desenvolver abordagens através de sucessivos processos de avaliação inclusivas a fim de identificar as dificuldades e propulsores de estímulos positivos para realização de atividades, e assim, poder incluí-las nas fases de design [Spiel et al 2017].

Muitas pesquisas [Satterfield e Fabri 2017] ressaltam a importância do DP para entender as reais necessidades dos usuários, principalmente crianças com transtornos do neurodesenvolvimento com comprometimentos cognitivos, como é o caso de muitas crianças com autismo. Dessa forma, [Frauenberger, Makhaeva e Spiel 2017] também afirmam que é importante promover atividades participativas combinando, reinterpretando e adaptando técnicas e ferramentas com base nas características dessas 
crianças. Essas pesquisas indicam que crianças com autismo podem participar ativamente do processo de design, fornecendo suas preferências e proporcionando aos designers a adaptação de modelos por meio dos preceitos de DI e DP.

Nesse mesmo seguimento, Wilson [Wilson et al 2017] descreve o envolvimento de crianças com autismo para registrar e expressar seus próprios interesses dentro de seus contextos de apoio - o lar e a sala de aula. Com isso, foi possível adquirir uma compreensão de como tecnologias de auto expressão levaram a uma comunicação verbal aprimorada através de modelagem de vídeo e engajamento em tarefas de classe.

Da mesma forma, utilizando uma abordagem para avaliação participativa com crianças com autismo Spiel [Spiel et al 2017] desenvolveu uma abordagem denominada PEACE (Avaliação Participativa com Crianças Autistas), a fim de abordar as limitações de avaliações definidas e conduzidas por pesquisadores no contexto de crianças com neuro-deficiência. Usando essa abordagem, foi possível incluir crianças com TEA em todas as fases de desenvolvimento da aplicação, incluindo o design.

Todos esses trabalhos enfatizam que a participação das pessoas a quem a tecnologia se destina, no caso crianças com autismo, nas diversas fases dos processos de design tendem a tornar as aplicações adaptáveis aos problemas individuais, podendo até possuir um grau de inteligência para tornar-se autônoma e colaborar também com crianças com deficiências similares [Benton et al 2012].

\section{Em Busca de Representações de Expressão}

O objetivo do Drawing Kids, baseado na técnica Brain Draw, é o reconhecimento das diferentes formas de representação que crianças com autismo realizam ao usarem essas ferramentas e assim, buscar identificar suas preferências. Dessa forma, foi necessária a incrementação de novas funcionalidades na aplicação para que possa ser possível uma avaliação mais ampla e detalhada.

Portanto, pesquisamos na literatura trabalhos que, baseadas no processo de DP e DI, desenvolveram técnicas para alcançar resultados significativos em busca do reconhecimento dessas representações. Sendo assim, adaptamos as funcionalidades do Drawing Kids (design inicial) baseadas em pesquisas voltadas para pessoas com autismo, preferencialmente crianças de 5 a 12 anos, buscando entender suas características particulares [Franceli et al 2018], [Dickstein-Fischer et al 2017], [Tang 2016], adaptando as tecnologias às suas necessidades incluindo os destinatários no processo de design [Michelle 2017], além de proporcionar uma visão sobre aspectos de preferências com respeito a aparência de interfaces [Melo et al 2017].

Portanto, o Drawing Kids, fundamentado nesses aspectos, buscou compor essas características acessíveis possibilitando maximizar a comunicação das crianças com autismo e assim auxiliar na avaliação e interpretação dos resultados, com o objetivo de reconhecer formas e critérios de representações.

\subsection{Planejamento e Preparação}

Para realizar o estudo e avaliação do aplicativo Drawing Kids foram realizadas parcerias com entidades educacionais que trabalham com crianças com autismo. Dessa forma, através de 2 instituições, na cidade de Manaus, Amazonas, realizamos os testes necessários para comprovar os objetivos propostos neste trabalho de pesquisa. 
As instituições disponibilizaram 1 professor, 1 pedagogo, 1 fisioterapeuta e 1 psicólogo que trabalham diretamente com as crianças. A equipe de avaliação foi composta por 4 avaliadores engenheiros de software. Além disso, os cuidadores (pais ou responsáveis pelos alunos) também foram convidados a participar durante o processo de avaliação de uso do aplicativo. A presença de todos os que convivem próximos às crianças se fazem necessário para auxílio e orientação. Isso se faz necessário para que através do feedback seja possível implementar tecnologias adaptativas para atender às necessidades específicas das crianças [Braz, Souza e Raposo 2014].

Os pais ou responsáveis assinaram o Termo de Consentimento Livre e Esclarecido (TCLE) autorizando a participação de seus filhos nas avaliações. Da mesma forma os profissionais da escola convidados para os testes, também assinaram o TCLE.

A escolha das crianças (12 no total) foi feita através do grau de severidade do autismo. Sendo assim, definimos os usuários com grau alto e médio de severidade, para que pudéssemos avaliar o nível de dificuldade do uso da ferramenta. Além disso, foi realizada uma entrevista prévia com os pais para coletar outras características do perfil das crianças como idade, sexo, se possui dificuldade linguística para que fosse necessário o auxílio direto nos testes, e experiência com smartphones ou tablets para saber se iriam conseguir utilizar o dispositivo adequadamente.

Tabela 1. Perfil dos Alunos Participantes.

\begin{tabular}{|c|c|c|c|c|}
\hline Crianças & $\begin{array}{c}\text { Grau de } \\
\text { Severidade }\end{array}$ & $\begin{array}{c}\text { Comunicação } \\
\text { Verbal }\end{array}$ & $\begin{array}{c}\text { Usa Dispositivos } \\
\text { Móveis }\end{array}$ & $\begin{array}{c}\text { Características } \\
\text { Associadas }\end{array}$ \\
\hline C1 & Médio & Sim & Não & \\
\hline C2 & Médio & Sim & Sim & \\
\hline C3 & Médio & Sim & Não & \\
\hline C4 & Alto & Não & Não & \\
\hline C5 & Médio & Sim & Sim & Locomoção \\
\hline C6 & Médio & Sim & Sim & \\
\hline C7 & Médio & Sim & Sim & Baixa Visão \\
\hline C8 & Alto & Não & Não & \\
\hline C9 & Médio & Sim & Sim & \\
\hline C10 & Médio & Sim & Sim & \\
\hline C11 & Médio & Sim & Sim & Baixa visão \\
\hline C12 & Alto & Não & Sim & \\
\hline
\end{tabular}

A Tabela 1 mostra que a maioria das crianças (9) tem grau de severidade médio de autismo, o que foi importante para uma comparação sobre o nível de usabilidade da aplicação. Outra informação importante é sobre a experiência com dispositivos móveis, na qual apenas 4 crianças não possuem habilidades. No entanto, os pais informaram que as crianças já utilizaram, mesmo que poucas vezes, smartphones ou tablets.

Os testes foram realizados nos laboratórios de informática das próprias instituições devido o ambiente ser conhecido e, portanto, o mais adequado para as crianças. Inicialmente, foram utilizados, para os testes, smartphones. No entanto, devido a 2 crianças participantes terem problemas de baixa visão os testes tiveram que ser realizados em 2 tablets utilizando uma imagem mais ampla.

\subsection{Avaliação do Estudo de Caso}

A implementação do Drawing Kids foi baseada no método de desenvolvimento ágil Scrum [Marçal 2007]. Da mesma forma, o design inicial (prototipação) passou por uma validação para identificação das falhas. Ainda na fase inicial de desenvolvimento, um teste de inspeção de usabilidade aplicado de acordo com as heurísticas de [Nielsen 
1994], que teve como objetivo verificar se o aplicativo desenvolvido está em um nível aceitável de usabilidade do ponto de vista de crianças com autismo. Os erros encontrados na inspeção de usabilidade foram considerados de baixa prioridade e foram corrigidos ainda durante a fase de desenvolvimento.

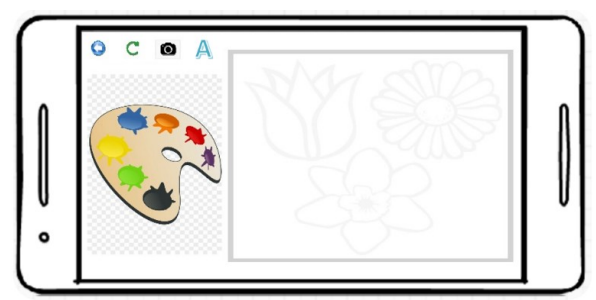

Figura 1. Tela de Atividades do Drawing Kids

Nos testes realizados com as crianças sugerimos apenas que eles cumprissem 4 tarefas na aplicação, sequencialmente para que fosse possível o acompanhamento dos avaliadores como mostra a Tabela 2 a seguir.

Tabela 2. Tarefas a Serem Realizadas no Drawing Kids

\begin{tabular}{|c|c|}
\hline $\mathbf{N}^{\mathbf{0}}$ & Descrição das Tarefas \\
\hline 01 & Escolher um objeto (figura) \\
\hline 02 & Cobrir e colorir o objeto \\
\hline 03 & Digitar um texto (nome) \\
\hline 04 & Refazer as atividades anteriores (a escolha) \\
\hline
\end{tabular}

Dessa forma, o estudo de caso teve ênfase numa avaliação qualitativa por meio de um Teste Exploratório [Bach 2004] que se trata de um teste para avaliar a percepção do usuário sobre um projeto de software. Assim, foi possível avaliar a qualidade de interação das crianças com a aplicação, mediante acompanhamento e observação dos pais e dos profissionais que, ao final dos testes, responderam uma Entrevista Semiestruturada sobre Experiência [Mason 2002] com os mesmos. A estratégia de combinar o Estudo Exploratório e a Entrevista Semiestruturada é de reforçar, por meio do feedback dos profissionais e cuidadores, o reconhecimento das informações sobre a predileção das crianças quanto aos recursos de design do aplicativo Drawing Kids.

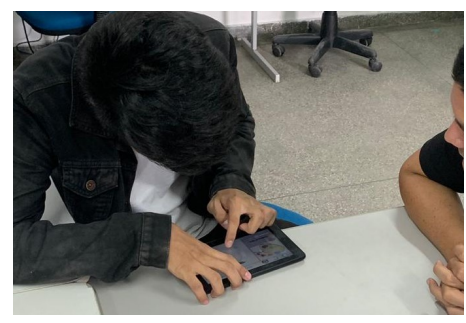

Figura 2. Aluno Realizando o Teste com o Drawing Kids

Portanto, a avaliação do estudo de caso foi realizada com as crianças interagindo com a aplicação através da mediação dos profissionais ou cuidadores que atendem as mesmas. Vale ressaltar que foi importante a intervenção para que fosse detalhado as tarefas as crianças e também para que os avaliadores pudessem acompanhar os passos das atividades realizadas, uma vez que algumas crianças possuem dificuldades linguísticas (Tabela 1). Todos os testes foram registrados (filmados e capturadas imagens) para posterior análise da equipe de avaliação pós-teste e discussão juntamente com os dados dos questionários e entrevistas. Os testes tiveram duração média de 22 minutos. 
IX Congresso Brasileiro de Informática na Educação (CBIE 2020)

Anais do XXXI Simpósio Brasileiro de Informática na Educação (SBIE 2020)

\subsection{Resultados}

Os principais dados obtidos dos testes com as crianças, ao utilizarem o aplicativo Drawing Kids, foram organizados na Tabela 3 como mostra a seguir.

Tabela 3. Resultado dos Testes Aplicados com as Crianças no Drawing Kids

\begin{tabular}{|c|c|c|c|c|c|}
\hline Criança & $\begin{array}{c}\text { Tempo } \\
\text { do Uso } \\
\text { (Minutos) }\end{array}$ & $\begin{array}{c}\text { Concluiu } \\
\text { a Tarefa }\end{array}$ & $\begin{array}{c}\text { Decidiu } \\
\text { Refazer }\end{array}$ & $\begin{array}{c}\text { Preferência } \\
\text { por cores }\end{array}$ & $\begin{array}{c}\text { Preferência } \\
\text { por figuras }\end{array}$ \\
\hline C1 & 22 & Não & Não & Não & Sim \\
\hline C2 & 25 & Sim & Sim & Sim & Sim \\
\hline C3 & 24 & Sim & Sim & Sim & Sim \\
\hline C4 & 31 & Não & Sim & Não & Sim \\
\hline C5 & 22 & Sim & Sim & Sim & Sim \\
\hline C6 & 19 & Sim & Sim & Sim & Sim \\
\hline C7 & 17 & Sim & Sim & Sim & Sim \\
\hline C8 & 30 & Sim & Sim & Sim & Não \\
\hline C9 & 18 & Sim & Sim & Sim & Sim \\
\hline C10 & 16 & Sim & Sim & Sim & Sim \\
\hline C11 & 15 & Sim & Não & Sim & Sim \\
\hline C12 & 32 & Sim & Sim & Sim & Não \\
\hline
\end{tabular}

Ao analisar a Tabela 3 notamos que os maiores tempos dos testes foram alcançados pelas crianças $\mathrm{C} 4, \mathrm{C} 8$ e $\mathrm{C} 12$, que são os alunos que tem problemas linguísticos. Dessa forma, a demora se dá pelo fato de precisarem de um tempo para compreender o que deveria ser feito no aplicativo (as tarefas), através do auxílio dos profissionais, como também fazerem o reconhecimento da interface do aplicativo através dos símbolos (botões). Além disso, possuem grau de severidade alto de autismo, o que necessitou de auxílio direto dos pais e profissionais para realizar os testes.

Apenas 2 crianças não conseguiram completar as atividades do teste (C1 e C4). Isso ocorreu pelo fato da criança $\mathrm{C} 1$ decidir parar espontaneamente a realização do teste. Nesse caso, um profissional informou que como a criança participou de uma sessão de fisioterapia e hidroginástica naquele dia a mesma estaria cansada. A criança $\mathrm{C} 4$ tentou realizar as tarefas, inclusive persistindo, com um tempo considerado alto (31 minutos). No entanto, não conseguia prender a atenção e demonstrando total inquietude.

A grande maioria (10 crianças) decidiram por refazer as atividades principalmente para que pudessem escolher outras cores, o que também reflete o resultado da coluna sobre (Preferência por cores) onde a maioria decidiu escolher cores prediletas para usar nas figuras. Algumas crianças escolheram as mesmas cores para refazer a atividade, trocando somente o local onde iria preencher a cor, o que só reforça as individualidades da mesma forma que em crianças sem transtornos. Coincidentemente, a maioria dos alunos escolheu cores mais claras para usar nas figuras. Isso talvez possa indicar um estado emocional mais reconfortante, mas requer uma investigação mais profunda nesse sentido, qual foge ao escopo deste trabalho.

A maioria também decidiu por escolher figuras de animais para colorir. $\mathrm{O}$ Drawing Kids possui imagens sobre animais, flores, objetos (comuns do dia a dia das crianças como lápis e livro). No entanto, estão dispostos sem agrupamento, e isso gerou comentários dos pais e profissionais. Segundo eles, as imagens deveriam estar dispostas em grupos separados por temas, e dentro de cada grupo disponibilizar as imagens ex: Grupo de Animais (Peixes, Cavalos, Cães, Gatos, etc). Dessa forma, as opções estariam mais compreensíveis para elas e estariam alinhadas a objetivos educacionais. Também sugeriram incluir mais figuras e seus grupos. 
Algumas crianças perguntaram sobre figuras de super-heróis e sobre personagens de desenhos animados. Os profissionais informaram que nas atividades escolares são envolvidos esses personagens, inclusive usam fantasias nas crianças, o que pode ter levado a esses questionamentos. Da mesma forma, perguntaram sobre os sons do aplicativo e sugeriram disponibilizar alguns de suas escolhas.

Durante os testes, foi perceptível que as características peculiares de algumas crianças se sobressaem em relação as outras. Apesar de serem diagnosticadas com o mesmo nível de severidade de autismo, algumas crianças conseguiram se adaptar e reconhecer as ações dos símbolos do aplicativo com mais facilidade. Por exemplo, o Drawing Kids possui um botão para capturar imagem da tela do aplicativo, o que não foi incluído nas tarefas a serem cumpridas. No entanto, algumas crianças reconheceram a função do botão e utilizaram para registrar seus desenhos.

Outra característica importante foi na atividade para digitar um texto. Nessa atividade, algumas crianças conseguiram digitar o próprio nome e de alguns familiares mais próximos, reconhecendo rapidamente o teclado e, inclusive, a disposição das letras. Contudo, nem todos identificaram o botão para inserir o texto e precisaram ser auxiliadas pelos especialistas.

O auxílio dos pais e profissionais também foi muito importante para realizar os testes, e como eles observaram as crianças utilizando o aplicativo, puderam expressar seus pontos de vista em relação aos resultados da pesquisa. Isso foi importante para que realmente houvesse a validação da aplicação.

Nas palavras dos profissionais: "A ideia é muito interessante e a estrutura desenvolvida para identificar elementos de percepções das crianças foi bem elaborada, mesmo que seja apenas algumas características como cores e imagens". Todos os profissionais e cuidadores relataram o design simples e autoexplicativo, apresentando padrões já conhecidos, o que traz o reconhecimento de funções da maioria dos botões.

De acordo com os pais: "Ferramentas computacionais, quando bem empregadas, sempre tem a capacidade de auxiliar de alguma forma as crianças, e o fato de a atividade dar a liberdade de escolher seus próprios objetos e poder organizálos de acordo com suas predileções dá autonomia as crianças".

\section{Conclusões e Perspectivas Futuras}

Ao utilizar o Drawing Kids, as crianças reproduziram suas percepções quanto à representação de objetos comuns, conhecidos por eles através de figuras e combinação de cores, e expressaram sua forma de compreender e representar essas imagens. As crianças tiveram a livre escolha de reproduzir as cores através das formas mais de uma vez, e com isso foi possível fazer anotações relevantes sobre cada uma de suas escolhas.

O reconhecimento de expressões e habilidades das crianças com autismo, mesmo que de forma genérica, contribui para os projetistas compreenderem e desenvolverem interfaces adequadas voltadas para esse público e, consequentemente, possibilitando uma melhor experiência de interação com artefatos computacionais.

Baseado no desenvolvimento do aplicativo e utilização do mesmo para capturar expressões, habilidades e preferências de objetos e organização espacial, as principais contribuições deste artigo estão relacionadas à participação das crianças om autismo, pois abrem novas possibilidades de design inclusivo e desenvolvimento de artefatos 
computacionais que irão auxiliá-las em atividades comuns de seus contextos educacionais e com isso poderem participar mais efetivamente dos espaços inclusivos.

Os resultados forneceram subsídios aos projetistas para repensarem as formas de incluírem crianças com autismo, antes alijadas do processo de design de tecnologia para elas mesmas, a participarem de processos de design de tecnologia, através de algumas adaptações no espaço físico e virtual, de forma a promover sua participação ativa não só no design de tecnologias, mas também em contextos educacionais inclusivos.

Por fim, assim que for possível, após os ajustes necessários no aplicativo, as atividades serão aplicadas envolvendo outras crianças com transtornos cognitivos e acrescentadas outras atividades que fomentem a expressão por desenhos.

\section{Agradecimentos}

Esta pesquisa, conforme previsto no Art. 48 do decreto $\mathrm{n}^{0} 6.008 / 2006$, foi parcialmente financiada pela Samsung Electrônica da Amazônia Ltda, nos termos da Lei Federal $\mathrm{n}^{\mathrm{o}}$ 8.387/1991, através de convênio no 003/2019, firmado com o ICOMP/UFAM.

A FAPEAM (Fundação de Amparo à Pesquisa do Estado do Amazonas), também pelo apoio financeiro dispensados a essa pesquisa.

\section{Referências}

APA. Associação Americana de Psiquiatria. (2014) DSM-V: Manual diagnóstico e estatístico de transtornos mentais. $5^{\text {a }}$ ed. Porto Alegre: Artmed. 992p.

Bach, J. (2004) "Exploratory Testing”. In The Testing Practitioner, Second ed., E. van Veenendaal Ed., Den Bosch: UTN Publishers, pp. 253-265.

Benton, L., Jhonson, H., Ashwin, E., Brosnan, M., and Grawemeyer, B. (2012) "Developing IDEAS: supporting children with autism within a participatory design team". In Proceedings of the SIGCHI Conference on Human Factors in Computing Systems (CHI '12). ACM, NY, USA, 2599-2608.

Braz, P., Souza, C. S. de, Raposo, A. (2014) "Design de Tecnologias Adaptáveis para Uso de Profissionais da Área de Autismo". Brazilian Symposium on Human Factors in Computing Systems. October 27-31, 2014, Foz do Iguaçu, PR, Brazil.

Britto, T. C. P., Pizzolato, E. B. (2016) "Gaia: uma proposta de um guia de recomendações de acessibilidade de interfaces web com foco em aspectos do autismo". V Congresso Brasileiro de Informática na Educação (CBIE 2016).

Dickstein-Fischer, L. A., Pereira, R. H., Gandomi, K. Y. Fathima, A. T. and Fischer, G. S. (2017) "Interactive Tracking for Robot-Assisted Autism Therapy". In Proceedings of the Companion of the 2017 ACM/IEEE International Conference on Human-Robot Interaction (HRI '17). ACM, New York, NY, USA, 107-108.

Franceli, L., Cibrian, J. M., Lizbeth, E., and Tentori, M. (2018) “A Step towards Identifying the Sound Preferences of Children with Autism". In Proceedings of the 12th EAI International Conference on Pervasive Computing Technologies for Healthcare (PervasiveHealth '18). ACM, New York, NY, USA, 158-167.

Frauenberger, C., Makhaeva, J., e Spiel, K. (2017) "Blending Methods: Developing Participatory Design Sessions for Autistic Children". In Proceedings of the 2017 Conference on Interaction Design and Children (IDC '17). ACM, New York, USA. 
IX Congresso Brasileiro de Informática na Educação (CBIE 2020)

Anais do XXXI Simpósio Brasileiro de Informática na Educação (SBIE 2020)

Ismail, R., Ibrahim, R. (2017) "PDEduGame: Towards Participatory Design Process for Educational Game Design in Primary School", IEEE Explor., pp. 1-6, 2017.

Marçal, A. et al. (2007) "Mapping CMMI project management process areas to SCRUM practices". In: Software Engineering Workshop. Proceedings.007. p. 13-22.

Mason, J. (2002) “Qualitative researching (2nd ed.)”. London: Sage.

Melo, A. M., M. Baranauskas, C. C., Soares, S. C. de M. (2008) "Design com Crianças: da Prática a um Modelo de Processo". Revista Brasileira de Informática na Educação, v. 16, n. 1, 43-55.

Melo, A. Santos, J., Rivero, L., and Barreto, R. (2017) "Searching for Preferences of Autistic Children to Support the Design of User Interfaces". In: Proceedings of the XVI Brazilian Symposium on Human Factors in Computing Systems (IHC 2017). ACM, New York, NY, USA, Article 45, 10 pages.

Michelle, T. C. Y. (2017) "Exploring inclusive design partnerships through an IDEA framework to support deaf or hard of hearing australian children in design process participation". In Proceedings of the 29th Australian Conference on Computer-Human Interaction (OZCHI '17). ACM, New York, NY, USA, 433-437.

Muller, M. M. J., Kuhn, S. (1993) "Participatory design”, Commun. ACM, vol. 36, no. 6, pp. 24-28, 1993.

Nielsen, J. (1994) "Heuristic evaluation". In Nielsen, J., and Mack, R. L. (Eds.), Usability Inspection Methods. New York. John Wiley \& Sons. 25-64.

Nunes, E. P. S., Luz, A. R., Lemos, E. M. (2016) “Approaches of Participatory Design in the Design Process of a Serious Game to Assist in the Learning of Hospitalized Children”. HCI, vol. 1, pp. 406-416, 2016.

Santana, F. C. B. G., Carvalho, L. R. de, Ribeiro, G. N. S., Aguiar, S. E. V., Santana, A. C. R. (2017) "SATA (Sistema de Acompanhamento do Tratamento de Autismo)". VI Congresso Brasileiro de Informática na Educação (CBIE 2017).

Satterfield, D., Fabri, M. (2017) "User Participatory Methods for Inclusive Design and Research in Autism: A Case Study in Teaching UX Design”. 186-197. 10.1007/978-3319-58634-2_15.

Silva, G. F. M., Salgado, L. C. C., Raposo, A. (2013) "Metáforas de Perspectivas Culturais na (re) definição de padrões de colaboração de um jogo de multi-toque para usuários com autismo". In: Proceedings of IHC'13, Brazilian Symposium on Human Factors in Computing Systems, SBC, Manaus, AM, Brazil.

Spiel, K., Malinverni, L., Good, J., Frauenberger, C. (2017) "Participatory Evaluation with Autistic Children". In Proceedings of the 2017 CHI Conference on Human Factors in Computing Systems (CHI '17). ACM, New York, NY, USA, 5755-5766.

Tang, T. Y. (2016) "Helping Neuro-typical Individuals to "Read" the Emotion of Children with Autism Spectrum Disorder: an Internet-of-Things Approach". In Proceedings of the The 15th International Conference on Interaction Design and Children (IDC '16). ACM, New York, NY, USA, 666-671.

Wilson, C. Brereton, M., Ploderer, B., Sitbon, L., and Saggers, B. (2017) "Digital Strategies for Supporting Strengths- and Interests-based Learning with Children with Autism". In Proceedings of the 19th International ACM SIGACCESS Conference on Computers and Accessibility (ASSETS '17). ACM, New York, NY, USA, 52-61. 